\title{
Estructura Dimensional de la Escala KidsLife, versión Colombia, para la Evaluación de Calidad de Vida en Discapacidad Intelectual
}

\section{Dimensional Structure of the KidsLife Scale, Colombia's version, for the Evaluation of Quality of Life in Intellectual Disability}

\author{
Adriana Mora Antó \\ Pontificia Universidad Javeriana Cali \\ Leonor Córdoba Andrade \\ Universidad del Tolima
}

\author{
Luisa Matilde Salamanca Duque \\ Universidad Autónoma de Manizales \\ Laura Elisabet Gómez Sánchez \\ Universidad de Oviedo
}

\begin{abstract}
La discusión en torno a la validez de la estructura factorial del constructo calidad de vida (CV) en el campo de la discapacidad intelectual ha planteado la necesidad de desarrollar nuevos estudios acerca de las dimensiones que lo componen. El objetivo de esta investigación fue analizar la estructura dimensional de la escala KidsLife, diseñada a partir del modelo de CV de Schalock y Verdugo Alonso (2007), compuesto por 8 factores. La escala fue aplicada a los cuidadores de 220 personas, con edades entre 4 y 21 años, diagnóstico de discapacidad intelectual y residentes en 3 ciudades de Colombia, seleccionada a partir de un muestreo por conveniencia. Se trató de un estudio instrumental, aplicando el modelo de ecuaciones estructurales para la realización del análisis factorial confirmatorio, análisis de fiabilidad compuesta, varianza media extractada y correlaciones múltiples R2. Se plantearon hipótesis de trabajo basadas en 5 diferentes modelos de CV. El modelo de 8 dimensiones interrelacionadas registró cargas factoriales que oscilaron entre 0,63 y 0,95 , correlaciones múltiples entre 0,34 y 0,81 , sin que se identificaran dimensiones de segundo orden. La escala registró una confiabilidad compuesta de 0,979 y una varianza media extractada superior al 50\%. Se concluye que el modelo propuesto de la escala KidsLife informa positivamente acerca de la CV de personas con discapacidad intelectual en las 3 ciudades colombianas. Se requieren nuevos estudios para identificar la posible existencia de factores de segundo orden.
\end{abstract}

Palabras clave: discapacidad intelectual, calidad de vida, análisis factorial confirmatorio, estudio instrumental, Escala KidsLife

\begin{abstract}
The discussion about the validity of the factor structure of the dimensions that constitute the quality of life (QOL) construct in the field of intellectual disability has highlighted the need to conduct new studies. The aim of this study was to analyze the dimensional structure of the KidsLife Scale, designed upon the basis of the 8-factor QOL model advanced by Schalock and Verdugo Alonso (2007). Using a convenience sample, the scale was administered to the caregivers of 220 people receiving support in specialized institutions. These caregivers' wards were between 4 and 21 years old, had been diagnosed with intellectual disability, and resided in 3 cities in Colombia. The study, of an instrumental nature, was conducted using structural equation modeling, confirmatory factor analysis, construct reliability analysis, average variance extracted, and multiple correlations R2. Working hypotheses based on 5 different models were analyzed. The model composed of 8 interrelated dimensions displayed factor loadings ranging from 0,63 to 0,95 , multiple correlations between 0,24 and 0,81 , a general reliability value of 0,979 , and an average variance extracted greater than $50 \%$, without any second-order dimensions being identified. It is concluded that the proposed KidsLife Scale model positively reports the QOL of people with intellectual disabilities in 3 Colombian cities. New studies are needed to identify the possible existence of second-order factors.
\end{abstract}

Keywords: intellectual disability, quality of life, confirmatory factor analysis, instrumental study, KidsLife Scale

Adriana Mora Antó, Departamento de Ciencias Sociales, Pontificia Universidad Javeriana Cali, Colombia; Luisa Matilde Salamanca Duque, Facultad de Salud, Universidad Autónoma de Manizales, Colombia; Leonor Córdoba Andrade, Instituto de Educación a Distancia, Universidad del Tolima, Ibagué, Colombia; Laura Elisabet Gómez Sánchez, Departamento de Psicología, Universidad de Oviedo, España.

Adriana Mora Antó es ahora profesional independiente, Cali, Colombia.

Este artículo está basado en el proyecto de investigación titulado "Validación de la Escala de Evaluación de Calidad de Vida para niños y adolescentes con discapacidad intelectual - KidsLife - en tres ciudades de Colombia", aprobado con código 457-063 de la Universidad Autónoma de Manizales, código 540120516 de la Universidad del Tolima y Acta DFHSC 82-3015 de 2015 del Comité de Ética de la Investigación de la Pontificia Universidad Javeriana Cali.

Las autoras colombianas agradecen a la Dra. Laura Elisabet Gómez Sánchez, autora de la Escala de Evaluación de Calidad de Vida para niños y adolescentes con discapacidad intelectual -KidsLife- y al Mg. Julián Andrés Tamayo Cardona, asesor estadístico por el procesamiento de datos.

La correspondencia relativa a este artículo debe ser dirigida a Adriana Mora Antó. E-mail: amora@javerianacali.edu.co 
La Calidad de Vida (CV, en adelante) se concibe como un constructo complejo que sirve de referente para el desarrollo de estudios válidos acerca del bienestar psicológico de las personas en general (Verdugo, Gómez, Arias \& Navas, 2012), ejerciendo un impacto positivo en la ejecución de programas de apoyo institucional en materia de educación o rehabilitación (Jenaro et al., 2005 citado en Simões et al., 2016; Verdugo, Navas, Gómez \& Schalock, 2012). Schalock y Verdugo (2007) se refieren a la CV como un estado deseado de bienestar personal de carácter multidimensional, que posee componentes objetivos y subjetivos, en los que se identifican propiedades éticas (universales) y propiedades ligadas a la cultura (émicas). Este concepto es útil para la redefinición de las funciones institucionales en materia de atención, dado que se obtienen valoraciones individualizadas del nivel de CV.

Diferentes estudios dan cuenta de la validez y utilidad del constructo CV basados en la aplicación de instrumentos de medición previamente validados. La validez se refiere al grado en el que el total de la evidencia acumulada apoya la interpretación de las puntuaciones de un test, de acuerdo con su propósito de medida, lo que implica hablar de distintos tipos de evidencia; por ejemplo, evidencia basada en la estructura interna del diseño, en el contenido de los ítems, en el procedimiento empleado para responder, en la relación de las puntuaciones con otras variables criterio o la evidencia acerca de las consecuencias del uso del test. Es por ello que los argumentos en favor de la validez de un test, pueden indicar la necesidad de refinar la definición del constructo que mide, la revisión de su diseño e incluso indicar la necesidad de nuevos estudios (American Educational Research Association, American Psychological Association \& National Council on Measurement in Education, 2014, p. 21). En resumen, la validez es un concepto unitario que alude a la calidad técnica del instrumento de medición.

En relación con la estructura interna de las escalas que se emplean para la medición de CV, Simões, Santos y Biscaia (2016), basados en el análisis de la versión en portugués de la Escala de Resultados Personales (POS) y la comparación de los modelos alternativos de la estructura factorial del constructo, refieren falta de consenso sobre el tema. El análisis factorial confirmatorio (AFC) de los datos provenientes de 1264 adultos con niveles leves o moderados de discapacidad intelectual, registró propiedades psicométricas adecuadas para las dimensiones de CV, destacando el modelo de Robert Schalock (ocho dimensiones) como el más robusto. Según este modelo, las dimensiones o factores que componen la CV, son: Inclusión Social, Autodeterminación, Bienestar Emocional, Bienestar Físico, Bienestar Material, Derechos, Desarrollo Personal y Relaciones Interpersonales (Schalock \& Verdugo, 2007).

En Brasil, el estudio realizado por Bredemeier, Peretti Wagner, Agranonik, Spalding Perez y Fleck (2014), basado en la aplicación del instrumento diseñado por la Organización Mundial de la Salud, para la evaluación de la calidad de vida de personas con discapacidad intelectual y física (WHOQOL-Dis-ID), aplicado a 156 personas con discapacidad física y 156 personas con discapacidad intelectual, corroboró la estructura factorial del modelo de ochos factores, al igual que la confiabilidad y la validez de los datos obtenidos, ratificando la utilidad del concepto. Por su parte, Guillén, Verdugo, Arias y Vicente (2015) basados en la Escala de Intensidad de Apoyos (SIS), aplicada a una muestra de 143 niños y adolescentes entre 5 y 16 años, encontraron adecuadas propiedades psicométricas en este instrumento, confirmando su confiabilidad y validez, al igual que el ajuste del modelo de ocho factores de CV a los datos obtenidos. Asimismo, Gómez, Alcedo, Arias et al. (2016) a partir de la aplicación de la versión original de la escala KidsLife, basada en el modelo de ocho factores de CV, encontraron que los dominios de CV eran relevantes y confirmaron la validez del constructo, al igual que la adecuada consistencia interna de la escala KidsLife (alfa de Cronbach, entre 0,812 y 0,949).

En Colombia, Córdoba, Henao y Verdugo (2016) analizaron la CV de una muestra de 602 adultos con discapacidad intelectual, mediante la aplicación de la escala INICO-FEAPS validada para Colombia, diseñada a partir del modelo de CV de Schalock y Verdugo (2007), además de proponer la realización de estudios basados en la aplicación de instrumentos de medición previamente validados. Otros estudios nacionales reiteran la necesidad de desarrollar nuevas investigaciones sobre el constructo CV en discapacidad (Arenas, Jaramillo, Marmolejo \& Cruz, 2013; Córdoba, Mora, Bedoya \& Verdugo, 2007; Padilla Muñoz, 2011).

Acorde a los planteamientos expuestos, esta investigación tuvo como objetivo analizar la estructura dimensional de la escala KidsLife para la evaluación de calidad de vida de niños y adolescentes con discapacidad intelectual, entre 4 y 21 años de edad, diseñada a partir del modelo clásico de Shalock y Verdugo (2007), además de disponer de una escala adaptada al contexto colombiano. 
Simões et al. (2016) anotan que, aunque se han aplicado diferentes modelos explicativos de CV, "generalmente no hay indicación de que los diferentes componentes tengan algún tipo de estructura jerárquica o secuencia causal" (Cummins, 2005, p. 701, citado en Simões et al., 2016), razón por la que no se tiene evidencia de los dominios centrales e indicadores que la explican y reconocen la necesidad de verificar el ajuste del modelo de CV en el campo de la discapacidad intelectual, constatando la multidimensionalidad del constructo. Además, refieren que "existen pocos estudios sobre la validación de su estructura factorial" (Gómez et al., 2011, citado en Simões et al., 2016) y muestran que, si bien existe una estructura de primer orden, se reconocen dos estructuras de segundo orden que reflejan la jerarquización de las dimensiones: la primera de ellas es la propuesta por Jenaro et al. (2005, citado en Simões et al., 2016), quienes identifican los siguientes factores de segundo orden: (1) Bienestar Personal (Bienestar Emocional, Relaciones Interpersonales, Autodeterminación y Desarrollo Personal), (2) Bienestar Físico y Bienestar Material, y (3) Empoderamiento (Derechos e Inclusión Social) y la segunda de ellas, es la estructura planteada por Schalock et al. (2005, citado en Simões et al., 2016), quienes refieren tres factores de segundo orden: (1) Independencia (Desarrollo Personal y Autodeterminación), (2) Participación Social (Relaciones Interpersonales, Inclusión Social y Derechos) y (3) Bienestar (Bienestar Emocional, Bienestar Físico y Bienestar Material).

En resumen, existe una estructura jerárquica o una secuencia causal entre las dimensiones de la CV, que corresponde a niveles interactivos y relacionales, que se ordenan de acuerdo con un grado de importancia específico que es relativo al contexto del que se extraen. De allí que sea importante profundizar en la comprensión del constructo y los modelos teóricos existentes, considerando la diversidad de contextos en que se producen los datos.

Respecto al tema, Gómez, Verdugo, Arias y Arias (2011) plantean que tales jerarquías pueden ser la consecuencia del tipo de instrumento aplicado, del proceso de validación y de las propiedades psicométricas del mismo o de la aplicación en muestras pequeñas y poco representativas. Estos investigadores reafirman que la CV está compuesta por ocho dominios de primer orden y enfatizan en el carácter multidimensional e interdependiente del concepto de CV; sin embargo, señalan que las explicaciones de segundo orden requieren nuevos estudios que confirmen su validez discriminante.

En este sentido, Wang et al. (2010) analizaron la estructura factorial y la naturaleza jerárquica del constructo CV, comparando el Modelo de Schalock (ocho factores de primer orden y tres factores de segundo orden: independencia, participación social y bienestar) y el Modelo de Jenaro (ocho factores de primer orden y tres de segundo orden: bienestar personal, bienestar físico y empoderamiento), basados en los datos de 769 personas con discapacidad intelectual moderada, provenientes de 15 países, concluyendo que el modelo Schalock fue el que mejor ajuste logró a los datos. Igualmente, encontraron un único factor de segundo orden al que denominaron "Constructo General de Calidad de Vida", pero también identificaron una dualidad en la formulación de los factores de segundo orden ("bienestar personal" y "empoderamiento"), dado que estaban igualmente saturados por el factor "autodeterminación". Estos investigadores plantean que los factores de primer orden pueden diferir entre países y culturas, en términos de cómo representan la calidad de vida, pero los factores de segundo orden pueden ser "universales", afirmaciones que requieren profundizarse. Por ello, se mantiene la discusión acerca de sí las dimensiones de CV se ordenan jerárquicamente o interactúan, a fin de explicar sus características.

Tanto Wang et al. (2010) como Isaacs et al. (2012) coinciden en que es necesario comprender mejor la naturaleza jerárquica de las dimensiones constitutivas del constructo $\mathrm{CV}$, recurriendo a la aplicación del modelo de ecuaciones estructurales (conocido como SEM, por su sigla en inglés), técnica empleada para estimar modelos de relaciones lineales entre las variables medidas y latentes (Kline, 1998 citado por Wang et al., 2010; Kline, 2005), dado que se pueden controlar las medidas que potencialmente representan, ya sea como producto de una diferencia actitudinal o de una respuesta distinta a los ítems de la escala. La aplicación de SEM debe basarse en hipótesis guiadas por la teoría acerca de la estructura del conjunto de indicadores de la escala; sin embargo, su aplicación también dependerá de que la muestra cumpla con los supuestos estadísticos de representatividad y distribución normalizada.

De lo anterior, se desprende que el análisis de la estructura dimensional de la escala KidsLife, diseñada a partir del modelo de Schalock, se realice evaluando el ajuste de los diferentes modelos teóricos de CV a los datos (Gómez et al., 2011), en función de las siguientes hipótesis de trabajo:

1. Modelo 1: La CV es un constructo unidimensional, que corresponde a un modelo explicativo nulo o no válido, opuesto al modelo teórico clásico. 
2. Modelo 2: La CV se compone de ocho factores correlacionados: Bienestar Emocional, Relaciones Interpersonales, Bienestar Material, Desarrollo Personal, Bienestar Físico, Autodeterminación, Inclusión Social y Derechos, correspondiente al modelo clásico de Schalock y Verdugo Alonso (2007).

3. Modelo 3: La CV se compone de ocho factores de primer orden (Bienestar Emocional, Relaciones Interpersonales, Bienestar Material, Desarrollo Personal, Bienestar Físico, Autodeterminación, Inclusión Social y Derechos) y uno de segundo orden, al que Wang et al. (2000) denominaron "Constructo Global de Calidad de Vida".

4. Modelo 4: La CV se compone de ocho factores de primer orden (Bienestar Emocional, Relaciones Interpersonales, Autodeterminación, Desarrollo Personal, Derechos, Inclusión Social, Bienestar Físico y Bienestar Material) y tres factores de segundo orden, compuestos en la siguiente forma: (a) Bienestar Personal (Relaciones Interpersonales, Bienestar Emocional, Desarrollo Personal y Autodeterminación); (b) Empoderamiento (Inclusión Social y Reconocimiento de Derechos); y (c) Bienestar Físico y Material (referido a estos dos dominios). Este modelo es derivado de la investigación de Jenaro et al. (2005).

5. Modelo 5: La CV se compone de ocho factores de primer orden (Desarrollo Personal, Autodeterminación, Relaciones Interpersonales, Inclusión Social, Derechos, Bienestar Emocional, Bienestar Físico y Bienestar Material) y tres de segundo orden: Independencia, Integración Social y Bienestar Personal. Corresponde al modelo identificado por Schalock et al. (2005).

El desarrollo de esta investigación contribuye a la evaluación contextualizada de los niveles particulares de CV en las tres ciudades colombianas, además, de que aporta a la evaluación de los programas de atención institucional y, eventualmente, a la ejecución de estudios transculturales, tal como lo proponen Wang, Schalock, Verdugo y Jenaro (2010). Además, el análisis de las dimensiones de CV ofrece una medida específica del grado de desarrollo personal de un individuo particular, que puede emplearse para el seguimiento de sus logros y para el trazado de metas institucionales (Schalock \& Verdugo, 2007).

\section{Método}

\section{Tipo de Estudio}

Se desarrolló un estudio de carácter instrumental (Carretero-Dios \& Pérez, 2007), siguiendo las directrices de la Comisión Internacional de Tests (Muñiz, Elosua \& Hambleton, 2013), para preparar el instrumento. Posteriormente, se aplicó un AFC para verificar la semejanza entre la estructura del modelo de ocho dimensiones de CV (Schalock \& Verdugo, 2007) y la estructura dimensional de la escala Kids-Life (Gómez et al., 2014).

\section{Participantes}

Se seleccionaron 220 cuidadores de personas con un diagnóstico de discapacidad intelectual, elegidos en instituciones de apoyo de tres ciudades de Colombia (Cali: 73 casos; Manizales: 74 casos e Ibagué: 73 casos). Participaron 10 instituciones (96,4\% eran entidades privadas y 3,6\% instituciones públicas), dos de ellas localizadas en Cali, dos en Manizales y seis en Ibagué. La escogencia de estas ciudades se debió a que se tenía acceso a ellas, dado que sus directivas aceptaron la invitación para participar en el estudio, por lo que se trató de un muestreo por conveniencia.

Inicialmente, se expuso el contenido de la propuesta investigativa ante las Directivas, Profesionales y Colaboradores de las instituciones e incluso a los Abogados Defensores de Familia, representantes legales de las personas con discapacidad, en aquellos casos en que se los que se había declarado a la persona en situación de orfandad, ajustándose a los lineamientos de la ley en Colombia. Posterior a la aprobación de las directivas, la profesional de apoyo institucional revisó las bases de datos, para identificar aquellos casos que cumplían con los requisitos de edad y diagnóstico de discapacidad intelectual de base, incluyendo otro tipo de diagnósticos médicos existentes.

Las características sociodemográficas de las personas con discapacidad se consignan en la Tabla 1. 
Tabla 1

Características Sociodemográficas de las Personas con Discapacidad $(N=220)$

\begin{tabular}{|c|c|c|c|}
\hline Variable & Categoría & $n$ & $\%$ \\
\hline Sexo & $\begin{array}{l}\text { Hombre } \\
\text { Mujer }\end{array}$ & $\begin{array}{r}133 \\
87\end{array}$ & $\begin{array}{l}60,5 \\
39,5\end{array}$ \\
\hline Nivel de discapacidad & $\begin{array}{l}\text { Leve } \\
\text { Moderada } \\
\text { Severa } \\
\text { Profunda } \\
\text { No sabe/no responde }\end{array}$ & $\begin{array}{l}26 \\
82 \\
86 \\
16 \\
10\end{array}$ & $\begin{array}{r}11,8 \\
37,3 \\
39,1 \\
7,3 \\
4,5\end{array}$ \\
\hline Nivel de necesidades de apoyo & $\begin{array}{l}\text { Limitado } \\
\text { Intermitente } \\
\text { Extenso } \\
\text { Generalizado }\end{array}$ & $\begin{array}{l}49 \\
61 \\
55 \\
55\end{array}$ & $\begin{array}{l}22,3 \\
27,7 \\
25,0 \\
25,0\end{array}$ \\
\hline Nivel de dependencia & $\begin{array}{l}\text { I: Moderado } \\
\text { II: Severo } \\
\text { III: Gran dependencia }\end{array}$ & $\begin{array}{l}94 \\
61 \\
65\end{array}$ & $\begin{array}{l}42,7 \\
27,7 \\
29,5\end{array}$ \\
\hline Cuenta con familia & $\begin{array}{l}\text { No (situación de orfandad) } \\
\text { Sí }\end{array}$ & $\begin{array}{r}46 \\
174\end{array}$ & $\begin{array}{l}20,9 \\
79,1\end{array}$ \\
\hline Tipo de escolarización & $\begin{array}{l}\text { Ninguno } \\
\text { Educación ordinaria } \\
\text { Educación especial } \\
\text { Educación inclusiva }\end{array}$ & $\begin{array}{r}33 \\
10 \\
171 \\
6\end{array}$ & $\begin{array}{r}15,0 \\
4,5 \\
77,7 \\
2,7\end{array}$ \\
\hline Estratificación socioeconómica (lugar de residencia) & $\begin{array}{l}\text { Estrato 1: bajo-bajo } \\
\text { Estrato 2: bajo } \\
\text { Estrato 3: medio-bajo } \\
\text { Estrato 4: medio } \\
\text { Estrato 5: medio-alto } \\
\text { Estrato 6: alto }\end{array}$ & $\begin{array}{r}35 \\
84 \\
49 \\
49 \\
2 \\
0\end{array}$ & $\begin{array}{r}16,0 \\
38,4 \\
22,4 \\
22,4 \\
0,9 \\
0,0\end{array}$ \\
\hline
\end{tabular}

En cuanto al diagnóstico de discapacidad, el $77,3 \%$ de ellos fue realizado por el médico general, inmediatamente después del nacimiento. En Colombia, el artículo 5 de la Ley 361 de 1997 (Congreso de Colombia, Ministerio de Educación Nacional, Ministerio de Trabajo y Seguridad Social, Ministerio de Salud \& Ministerio de Transporte, 1997) establece que las personas con limitación deberán aparecer calificadas como tales en el carné de afiliado al Sistema de Seguridad en Salud, lo que se verifica mediante el diagnóstico médico; además, los médicos generales pueden intervenir en la atención de parto, atención del recién nacido y la detección temprana de alteraciones del crecimiento y desarrollo en menores de 10 años, información que se consigna en las historias clínicas de las entidades prestadoras de servicios en salud (Resolución 1441 de 2013 del Ministerio de Salud y Protección Social).

En lo que se refiere a la estratificación socioeconómica, el 79,1\% de los discapacitados residía en los estratos 1, 2, 3 y 4, en tanto que solo el 0,9\% vivía en el estrato 5. El 20,9\% de los discapacitados que había sido declarado en situación de orfandad, residía en la sede de la entidad, por lo que se les asignó el estrato 4. Se aclara que la estratificación socioeconómica de las residencias colombianas se basa en el sistema que provee el Estado (DANE, 2018), en el que se establece que los estratos 1, 2 y 3 albergan a los usuarios con bajos recursos económicos y beneficiarios de subsidios; los estratos 5 y 6 corresponden a los usuarios con altos recursos, en tanto que el estrato 4 no es beneficiario de subsidios, ni debe pagar sobrecostos por los servicios públicos que recibe.

Los informantes fueron escogidos por el profesional de apoyo institucional, de modo que se asegurase de su conocimiento acerca de las condiciones de vida de la persona discapacitada. El 41,8\% de ellos, fue personal de apoyo (director(a) escolar y/o orientador(a) escolar); el 30,0\% sus madres, el 12,3\% sus profesores, el 10,5\% los profesionales de apoyo (fonoaudiólogo(a), psicólogo(a), enfermero(a) o trabajador(a) social) y el 5,5\% correspondió a otros familiares diferentes a la madre. De ellos, el 89,1\% fueron mujeres y el 
10,9\%, hombres. Solo el $0,5 \%$ dijo mantener contacto una vez a la semana con la persona discapacitada, mientras el 59,1\% mantenía contacto permanente y el 40,5\% lo tenía varias veces por semana.

\section{Instrumentos}

Escala KidsLife. Se adaptó la versión original de la Escala KidsLife (Gómez, Alcedo, Verdugo et al., 2016), cuyo propósito es evaluar la CV individual percibida en personas entre 4 y 21 años de edad, con discapacidad intelectual/múltiples y profundas.

La versión original está compuesta por 156 reactivos, distribuidos en ocho dimensiones: Inclusión Social (IS), Autodeterminación (AU), Bienestar Emocional (BE), Bienestar Físico (BF), Bienestar Material (BM), Derechos (DE), Desarrollo Personal (DP) y Relaciones Interpersonales (RI), acordes al modelo de Schalock y Verdugo. La versión adaptada tiene 96 ítems que deben ser respondidos por una persona que conozca lo suficientemente bien al discapacitado, al menos, por un periodo de tiempo igual o superior a seis meses; esta versión mostró una consistencia interna adecuada (Alfa de Cronbach entre 0.81 [derechos] y 0.94 [desarrollo personal]) y se confirmó su validez de constructo mediante la aplicación del AFC (Gómez, Alcedo, Arias et al., 2016).

La versión original de KidsLife también fue adaptada para Colombia, registrando una consistencia interna adecuada (Alfa de Cronbach de 0.95, escala total), según lo reportado por Córdoba-Andrade; Mora Anto y Salamanca Duque (2019). Este proceso de adaptación se realizó en tres fases: Fase 1: Adaptación contextual de la Escala, Fase 2: Validación por jueces expertos y Fase 3: Estudio piloto, tal como lo refieren Salamanca-Duque, Mora-Anto y Córdoba-Andrade (2019).

En la fase 1 "Adaptación contextual de la Escala", se revisaron en forma preliminar, los 156 ítems por parte del equipo de investigadoras, considerando como criterios la importancia, influencia, observancia y adecuación gramatical. El criterio "importancia" aludía al valor o interés que la situación descrita por el ítem, tenía para evaluar la dimensión de CV en el contexto colombiano; el criterio "influencia" se refería a la pertinencia que el indicador tenía para valorar la dimensión; la "observancia" exploraba la posibilidad de que el ítem fuera visible por una persona externa y la "adecuación gramatical" el ajuste del enunciado del ítem, en cuanto a su estructura y comprensión idiomática en Colombia. Arribas (2006) explica que además de la equivalencia técnica, es importante que una escala de medición asegure la comprensión del lenguaje, a fin de mantener su paridad cultural, conservar el mismo significado y evaluar conceptos acordes a los propuestos en la versión original.

En la fase 2 "Validación por Jueces Expertos", se realizó con el apoyo de siete jueces, quienes nuevamente valoraron los ítems de acuerdo con los anteriores criterios. Formaron parte del equipo de jueces, dos fisioterapeutas expertos en discapacidad y rehabilitación; dos profesionales en el campo de la lingüística y tres psicólogos con experiencia en apoyo a la discapacidad, intervención con familias y discapacidad e investigación psicométrica; estas personas revisaron los ítems en forma independiente e hicieron la respectiva valoración, calificando con una escala de 1 a 4 puntos, donde 1 correspondía a totalmente en desacuerdo y 4 a totalmente de acuerdo.

Seguidamente, se creó la base de datos con las calificaciones de los jueces y se analizó la concordancia entre ellos, mediante el cálculo de coeficientes de concordancia ponderados (BWN: Bangdiwala \& Bryan, 1987), registrando valores superiores a 0,70 en los criterios de importancia, influencia y observancia. Los coeficientes más altos se obtuvieron en la dimensión Autodeterminación (importancia: $\mathrm{BWN}=0,858$; influencia: $\mathrm{BWN}=0,858$ y observancia: $\mathrm{BWN}=0,885)$. Posteriormente, se revisó la adecuación gramatical de los ítems, conforme a las sugerencias de los jueces y disponer de la versión piloto de la Escala.

De los 156 ítems de la escala original, se eliminaron 60 ítems (7 por baja capacidad de discriminación, 2 por baja correlación ítem/dimensión, 16 por ambas razones y 35 porque los informantes reportaron una escasa comprensión o porque las investigadoras consideraron que eran redundantes o generaban una posible deseabilidad social al responderse); además, se modificaron 65 ítems (20 ítems por baja capacidad de discriminación, 2 por baja correlación ítem/dimensión, 9 por baja capacidad de discriminación y baja correlación ítem/dimensión y 34 por bajo nivel de claridad y comprensión).

En la fase 3 "Estudio Piloto", se aplicó la versión adaptada de la escala compuesta por 96 reactivos, distribuidos en las dimensiones de CV (12 ítems en cada una). A modo de ilustración, se presenta un ítemtipo para cada una de las dimensiones: IS- Inclusión Social ("La persona va de vacaciones a lugares diferentes a donde vive (p.ej. casa de familiares, de conocidos de la familia, parques temáticos y/o hoteles) 
participando en las actividades e interactuando con otras personas"); AU- Autodeterminación ("Las personas que le proporcionan apoyos toman medidas específicas (p.ej. consultar sus preferencias y opiniones) para permitir que (nombre de la persona evaluada) haga elecciones por sí mismo/misma (p.ej. Actividades diarias, elecciones de lugares y personas)"; BE- Bienestar Emocional ("En el centro, se trata a (nombre de la persona) con cariño y afecto apropiado, cuando lo necesita"); BF- Bienestar Físico ("La persona toma la cantidad de comida y líquidos necesaria, para mantener un buen estado de salud"); BMBienestar Material ("La persona cuenta con las ayudas técnicas apropiadas para desarrollar al máximo su autonomía (p.ej. órtesis, bastón, silla de ruedas, audífonos, lentes, tableros de comunicación, tabletas)"); DE- Derechos ("Las personas que le proporcionan apoyos cuentan con formación específica sobre ética y respeto por los derechos de las personas con discapacidad"); DP- Desarrollo Personal ("Las personas que le proporcionan apoyos toman medidas específicas para mantener las habilidades y capacidades de (nombre de la persona)) y RI- Relaciones Interpersonales ("En el centro, se diseñan actividades que facilitan las interacciones sociales de (nombre de la persona) con los demás compañeros/as)".

$\mathrm{Al}$ igual que la versión original, se empleó una escala ordinal con cuatro opciones (1: nunca, 2: algunas veces, 3: frecuentemente y 4: siempre), de forma que un mayor puntaje correspondía a un mejor nivel de CV.

El estudio piloto se adelantó con 30 informantes que hicieron observaciones respecto a la claridad o la dificultad para comprender los enunciados. La escala se aplicó en forma directa por estudiantes universitarios, monitores de investigación con formación de pregrado en Psicología y Fisioterapia, además de una de las investigadoras en su respectiva ciudad. Con anterioridad, el equipo de investigadoras revisó, discutió y acordó el procedimiento para aplicar la escala, instrucciones que fueron explicadas a los monitores de la investigación en cada ciudad.

Posteriormente, se realizó un análisis exploratorio inicial y la estimación de la consistencia interna de la escala (Alfa de Cronbach superior a 0,80 para todas las dimensiones, con excepción de bienestar emocional [0,61], bienestar físico [0,75] y derechos [0.78]). Por último, se envió la versión colombiana a uno de los autores de la Escala, con el propósito de constatar que los conceptos evaluados mantuvieran correspondencia con la versión original.

Información sociodemográfica. Se recogió información sociodemográfica acerca de la persona evaluada (lugar de nacimiento, edad, tipo de escolarización, lugar de residencia actual, barrio, estratificación socioeconómica de la residencia) además de su nivel de discapacidad intelectual, profesional que lo diagnosticó, nivel de necesidades de apoyo requerido, nivel de dependencia reconocido y otros diagnósticos médicos confirmados. Se recogieron datos del informante principal relacionados con su conocimiento de la persona evaluada e información acerca del tipo de escolaridad brindada por la institución.

\section{Procedimiento}

Se elaboró una declaración de consentimiento informado, acogiéndose a los principios que regulan la investigación con seres humanos, orientaciones éticas establecidas por la Declaración de Helsinki (Asociación Médica Mundial, 2017) y la resolución 8430 de 1993 del Ministerio de Salud de Colombia. El contenido de este consentimiento fue aprobado por el Comité de Bioética de la Universidad Autónoma de Manizales (Acta Nº54 del 10 de febrero de 2016) y el Comité de Ética de la Investigación de la Facultad de Humanidades y Ciencias Sociales de la Pontificia Universidad Javeriana Cali (Acta DFHSC 82-3015 de junio de 2015).

Posteriormente, se presentó la propuesta de investigación a los padres de familia y/o acudientes, explicando los objetivos del estudio, los requisitos para participar, el aporte que tenía para la institución en cuanto a disponer de un instrumento adaptado para el contexto local y la posibilidad de acompañamiento psicoeducativo al informante, en el momento de responder a la escala. Esta presentación permitió constatar el interés de los padres de familia en colaborar con el estudio.

La aplicación de los cuestionarios se realizó de forma individual, al interior de las instalaciones de la entidad y/o fundación participante. Cada cuestionario fue resuelto con el apoyo de un integrante del equipo investigador, con un promedio de una hora de duración. Cuando la informante era la madre, se explicaba la declaración de consentimiento en presencia de la persona con discapacidad, reiterando el anonimato en la participación. Cuando no se contaba con la familia, se obtuvo la aprobación escrita del abogado Defensor de Familia y la profesional de apoyo institucional, previa presentación del acta de consentimiento. 
Se explicó y firmó la declaración de consentimiento, aclarando los objetivos del estudio, las condiciones de la participación, el riesgo mínimo para los participantes, la confidencialidad en los datos personales y el beneficio para la entidad en el proceso de identificar las condiciones de CV de las personas. Al finalizar, se entregó una copia firmada del documento, reiterando que el nombre de las personas no sería expuesto.

\section{Análisis de Datos}

Inicialmente, se especificaron los cinco modelos a evaluar, basándose en el sustento teórico:

Modelo 1: La calidad de vida es un constructo unidimensional, en el que el número de parámetros estimados, fueron 47 (24 varianzas de los errores de medida, 23 efectos directos del factor sobre los indicadores y una varianza de la variable latente).

Modelo 2: La calidad de vida se compone de 8 factores correlacionados (modelo de Schalock y Verdugo, 2007): bienestar emocional, relaciones interpersonales, bienestar material, desarrollo personal, bienestar físico, autodeterminación, inclusión social y derechos, en el que el número de parámetros estimados, fueron 76 (24 varianzas de los errores de medida, 16 efectos directos de los factores sobre los indicadores, 28 covarianzas entre los factores y 8 varianzas de las variables latentes).

Modelo 3: La calidad de vida se compone de 8 factores de primer orden y uno de segundo orden: Factor general de Calidad de vida, para el que el número de parámetros estimados fueron 56 (24 varianzas de los errores de medida, 16 efectos directos de las variables endógenas sobre los indicadores, 8 errores de medida de las variables latentes endógenas, 7 efectos directos de la variable exógena sobre las endógenas y una varianza de la variable exógena).

Modelo 4: La calidad de vida se compone de 8 factores de primer orden y tres de segundo orden: Bienestar Personal (bienestar emocional, relaciones interpersonales, desarrollo personal, autodeterminación), Empoderamiento (inclusión social, derechos) y Bienestar Físico y Material (bienestar físico, bienestar material), donde el número de parámetros a estimar, eran 59 (24 varianzas de los errores de medida, 16 efectos directos de las variables endógenas sobre los indicadores, 8 errores de medida de las variables latentes endógenas, 5 efectos directos de las tres variables exógenas sobre las endógenas, 3 varianzas de las variables exógenas y 3 covarianzas entre las variables exógenas).

Modelo 5: La calidad de vida se compone de 8 factores de primer orden y tres de segundo orden: Independencia (desarrollo personal y autodeterminación), Integración Social (relaciones interpersonales, inclusión social, derechos) y Bienestar Personal (bienestar emocional, bienestar físico, bienestar material). En este modelo, al igual que el anterior, el número de parámetros estimados, fueron 59 (24 varianzas de los errores de medida, 16 efectos directos de las variables endógenas sobre los indicadores, 8 errores de medida de las variables latentes endógenas, 5 efectos directos de las tres variables exógenas sobre las endógenas, 3 varianzas de las variables exógenas y 3 covarianzas entre las variables exógenas).

Previa aplicación del AFC, fue necesario preparar los datos en razón a que los Métodos de Ecuaciones Estructurales (SEM, por sus siglas en inglés) parten de varios supuestos sobre la distribución de ellos y su no cumplimiento afecta la eficiencia de los estimadores, lo que puede conducir a que el AFC no encuentre la solución indicada (Arias, 2008; Cupani, 2012; Hair et al., 2006). Todos estos análisis se realizaron a través del programa estadístico R Core Team (2017), previo a los análisis de fiabilidad de la escala.

Para la comprobación de los supuestos de SEM, se examinaron todas las variables en la siguiente forma: Análisis de las variables relevantes de los modelos; medida ordinal de las variables; asignación de cuatro valores por cada variable; existencia de normalidad en la distribución de los datos, a partir de la asimetría y la curtosis de las variables agrupadas en parcels (George \& Mallery, 2001), al igual que la aplicación de la prueba de Shapiro Wilk y las pruebas Mardia (5119,74, $p<0,001)$, Henze-Zirkler, (1,098, $p<0,001)$ y Royston, $(1097,49, p<0,001)$, con las que se determinó que no existía normalidad multivariada; análisis de los outliers o valores atípicos de los datos univariados y multivariados, mediante la distancia Robusta de Mahalanobis al Cuadrado (57.81) y la identificación de casos atípicos; análisis de la homocedasticidad (prueba de Levene, $F=16,763, p<0,000$ ), con la que se rechazó la hipótesis de igualdad de varianzas de las variables, al nivel de significación del $5 \%$; conteo de los datos perdidos (0,1\%). También se verificó la linealidad de los datos, recurriendo a la representación gráfica de las diferentes combinaciones 
posibles de los parcels agrupados por dimensión (en este caso, los parcels 1 y 3 de la dimensión BF y los parcels 1 y 2 de la dimensión DE, mostraron falta de linealidad); evaluación de la multicolinealidad entre los parcels, registrando dos coeficientes de correlación altos $(r=0,776$, entre el P2 y el P3 de la dimensión DP y $r=0,762$, entre el P1 y el P3 de la dimensión BM) y la correlación múltiple más alta $\left(r_{\mathrm{xy}}=0,74\right.$, para la dimensión DP, valor que se consideró adecuado, dado que fue $r_{\mathrm{xy}}>0,90$ ). Se verificó el número mínimo de observaciones $(\mathrm{n}=220)$, que superó ampliamente la recomendación de disponer de más de 120 observaciones, o de 5 observaciones por cada parcel (Breckler, 1990; Kline, 2005) y se analizó el número de indicadores por cada variable latente, para evitar problemas de identificación y convergencia entre las variables o valores enormes de $X^{2}$ que imposibilitaran el ajuste de los datos (Bentler \& Chu, 1987; Lomax, 1982).

Se crearon tres parcels por cada variable latente (dimensión), para un total de 24 parcels. Dado el alto número de ítems de la escala (96 en total, 12 por cada dimensión de CV), se agruparon en 24 parcels (3 parcels para cada dimensión, compuestos por cuatro ítems). La agrupación de ítems en parcels se realizó con base en su inclinación opuesta, es decir, se unieron ítems que presentaban asimetría en direcciones contrarias (Holt, 2004; Temperaal, Schim, \& Gijselaers, 2007), con el propósito de mejorar las cualidades psicométricas y reducir el número de variables observadas, verificando su unidimensionalidad. Los tres parcels $(\mathrm{P})$ agrupados en cada dimensión, fueron:

Inclusión Social (IS): P1_1 (ítems 10, 4, 11 y 7); P1_2 (ítems 6, 5, 8 y 1) y P1_3 (ítems 2, 12, 9 y 3).

Autodeterminación (AU): P2_1(ítems 8, 12, 7 y 11); P2_2 (ítems 6, 1, 9 y 5) y P2_3 (ítems 10, 2, 4 y 3).

Bienestar Emocional (BE): P3_1 (ítems 2, 3, 8 y 7); P3_2 (ítems 1, 5, 12 y 9) y P3_3 (ítems 4, 10, 11 y 6).

Bienestar Físico (BF): P4_1 (ítems 6, 9, 5 y 3); P4_2 (ítems 10, 8, 12 y 4) y P4_3 (ítems 7, 2, 11 y 1).

Bienestar Material (BM): P5_1 (ítems 2, 7, 5 y 9), P5_2 (ítems 12, 10, 6 y 11) y P5_3 (ítems 1, 3, 4 y 8).

Derechos (DE): P6_1 (ítems 1, 2, 9 y 8), P6_2 (ítems 11, 7, 12 y 10) y P6_3 (ítems 4, 5, 3 y 6).

Desarrollo Personal (DP): P7_1 (ítems 9, 4, 2 y 5); P7_2 (ítems 1, 12, 3 y 11) y P7_3 (ítems 10, 7, 8 y 6).

Relaciones Interpersonales (RP): P8_1 (ítems 7, 8, 12 y 4); P8_2 (ítems 9, 11, 6 y 5) y P8_3 (ítems 1, 10, 2 y 3).

De acuerdo con las pruebas de unidimensionalidad, todos los eigenvalues superaron el valor de 1 , el eigenvalue más bajo fue de 1,70 y el valor máximo de 2,52; también se observó que el primer factor (parcels) explicaba más del $40 \%$ de la varianza total, en todos los casos; y respecto a las comunalidades, se obtuvieron valores superiores a 0,5 en todos los casos. Por último, el porcentaje de variación entre la varianza del primer factor y el segundo factor fue superior al $40 \%$, en todos los parcels.

Debido a que los datos no se distribuyeron normalmente, se aplicó el método de estimación de parámetros denominado Mínimos Cuadrados Ponderados Diagonalizados (DWLS). Una de sus ventajas es que permite introducir en los análisis variables ordinales, dicotómicas y continuas que no se ajusten a criterios de normalidad (George \& Mallery, 2001), tal como ocurría con las variables ordinales de la escala (Gómez et al., 2014), facilitando la comprobación de los supuestos del AFC. Con la aplicación del DWLS se corrigen los problemas con el supuesto de normalidad y homocedasticidad, utilizando un método de distribución libre asintótica que permite estimar los parámetros; sin embargo, el valor del estadístico Chicuadrado $\left(X^{2}\right)$ que proporciona será más preciso, cuando más grande sea la muestra.

En lo concerniente a los índices de bondad de ajuste para evaluación de los modelos teóricos al conjunto de datos, se determinaron medidas absolutas de ajuste, medidas increméntales y medidas de ajuste parsimonioso (Bentler, 1992; Brown, 2006; Hu \& Bentler, 1999; Kline, 2005; Ruiz, Pardo \& San Martin, 2010):

El índice Chi-cuadrado $\left(X^{2}\right)$ corregido de Satorra-Bentler que es apropiado cuando no hay normalidad en la distribución de los datos; puede decirse que valores grandes de $X^{2}$ corresponderán a un ajuste deficiente y valores pequeños a un mejor ajuste, de allí que también se analice la magnitud de su valor.

El índice SRMR (Residuo Cuadrático Medio de Raíz Estandarizado), que se define como la diferencia estandarizada entre la correlación observada y la correlación predicha, cuyo rango de variación oscila entre 0 y 1 . Dado que es una medida de ajuste absoluto, un valor de cero indica un ajuste perfecto; un valor inferior a 0,05 un buen ajuste y un valor superior a 0,05 un ajuste aceptable. SRMR es sensible a la complejidad del modelo, pero a diferencia de $X^{2}$ es relativamente insensible al tamaño de la muestra.

Como medida de ajuste parsimonioso, se adoptó el índice RMSEA ('Error Cuadrático Medio de Aproximación o Root Mean Square Error of Aproximation'), cuyo valor ha de ser menor que 0,05 y prueba el 
grado en que el modelo se ajusta razonablemente bien en la población; valores entre 0,05 y 0,08 indican un ajuste razonable, valores entre 0,08 y 0,10 un ajuste mediocre y valores por encima de 0,10 , muestran un claro desajuste.

Como medidas incrementales de ajuste, el CFI (Índice comparativo de Bentler-Bonett) cuyo rango de variación está entre 0 y 1 ; un valor por encima de 0,90 muestra un ajuste adecuado del modelo y un valor cercano a 0,95 o mayor indica un ajuste razonablemente bueno del modelo. Y el TLI (Índice de Tucker Lewis o Índice no normalizado de ajuste), cuya escala de medida no está determinada, razón por la que sus valores se ubican entre 0 y 1 , pero se considera que un valor superior a 0,95 revela un buen ajuste.

Posteriormente, se evaluó la fiabilidad y validez de los indicadores de la escala KidsLife. Se calculó la fiabilidad compuesta de cada variable latente, analizando la consistencia interna de los indicadores dentro de un mismo factor; valor que debe estar por encima de 0,60. De forma complementaria, se estimó la varianza media extractada que mide la cantidad de varianza de los indicadores que es capturada por cada constructo, comparada con la que es capturada por el error de medida, indicador que debe ubicarse por encima de 0,50, para considerarse fiable. Igualmente, se determinaron los niveles de consistencia interna de las dimensiones evaluadas por la Escala KidsLife, mediante la aplicación del coeficiente Alfa de Cronbach, cuyo valor óptimo suele ser mayor o igual a 0,8 .

\section{Resultados}

En las figuras 1, 2, 3 y 4, se identifican los factores de primer (variables endógenas) y de segundo orden (variables exógenas), dependiendo de si se trata de un modelo multidimensional (M2, M3 y M5) o unidimensional (M1); también los 24 parcels o indicadores, siguiendo la notación $\mathrm{Pn} \_k$, donde $\mathrm{n}$ representa el número de la dimensión o factor de primer orden al que pertenecen cada parcels y $\mathrm{k}$ el número del parcels. Los factores de primer y segundo orden se simbolizan con óvalos y los parcels con rectángulos.

Estas figuras muestran los parámetros estimados para cada modelo, incluyendo las covarianzas entre

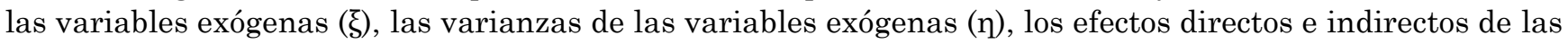
variables exógenas sobre las endógenas $(\mathrm{Y})$, los errores de medida de las variables latentes endógenas (s), los efectos directos e indirectos de las variables endógenas sobre los indicadores $(\lambda)$ y la varianzas de los errores de medida de los indicadores para la predicción del modelo $(\theta)$. El sistema de notación empleado (alfabeto griego), corresponde a la clave que se empleó para estimar los parámetros de las matrices de correlaciones, incluyendo las variables exógenas y endógenas y los respectivos errores de medida (sistema de ecuaciones estructurales de cada modelo).

En cuanto al contenido de las figuras, las relaciones entre variables se expresan mediante flechas; una flecha de doble punta y línea curva representa una covarianza o correlación entre los datos, y una flecha unidireccional con línea recta corresponde a un efecto directo de una variable sobre otra. Se utilizan líneas continuas para expresar el efecto directo de las cargas factoriales de una variable y líneas discontinuas para expresar su efecto indirecto. Un efecto directo describe la relación entre la variable latente y la medida (indicador) o entre dos variables latentes, mientras que un efecto indirecto se refiere a una relación entre una variable latente independiente y una variable latente dependiente, cuando su efecto esta mediado por otra variable latente externa al modelo. A lo largo de esta presentación de resultados, los efectos de las variables o indicadores equivalen a cargas o saturaciones factoriales, por lo tanto, se utilizan como sinónimos; de igual forma, cuando se habla de covarianzas entre factores y correlaciones múltiples.

La Figura 1 corresponde a la solución del Modelo 1 (unidimensional). Se encontró que todos los coeficientes fueron significativamente diferentes de cero $(p<0,05)$. Los errores de predicción oscilaron entre 0,43 (P2_3) y 0,85 (P4_3). Las saturaciones factoriales oscilaron entre 0,38 (P4_3) y 0,75 (P2_1, P2_3), encontrándose que 22 de 24 indicadores registraron una carga factorial por encima de 0,5 . Los coeficientes de correlación múltiple $\left(R^{2}\right)$ para cada indicador fluctuaron entre 0,14 y 0,56 , es decir, solo nueve superaron el $50 \%$ de la proporción de varianza de las variables observadas, siendo explicada por un único factor latente. Estos resultados mostraron que la solución unifactorial no era la adecuada, dado que no reproducía la matriz de covarianza del modelo. 


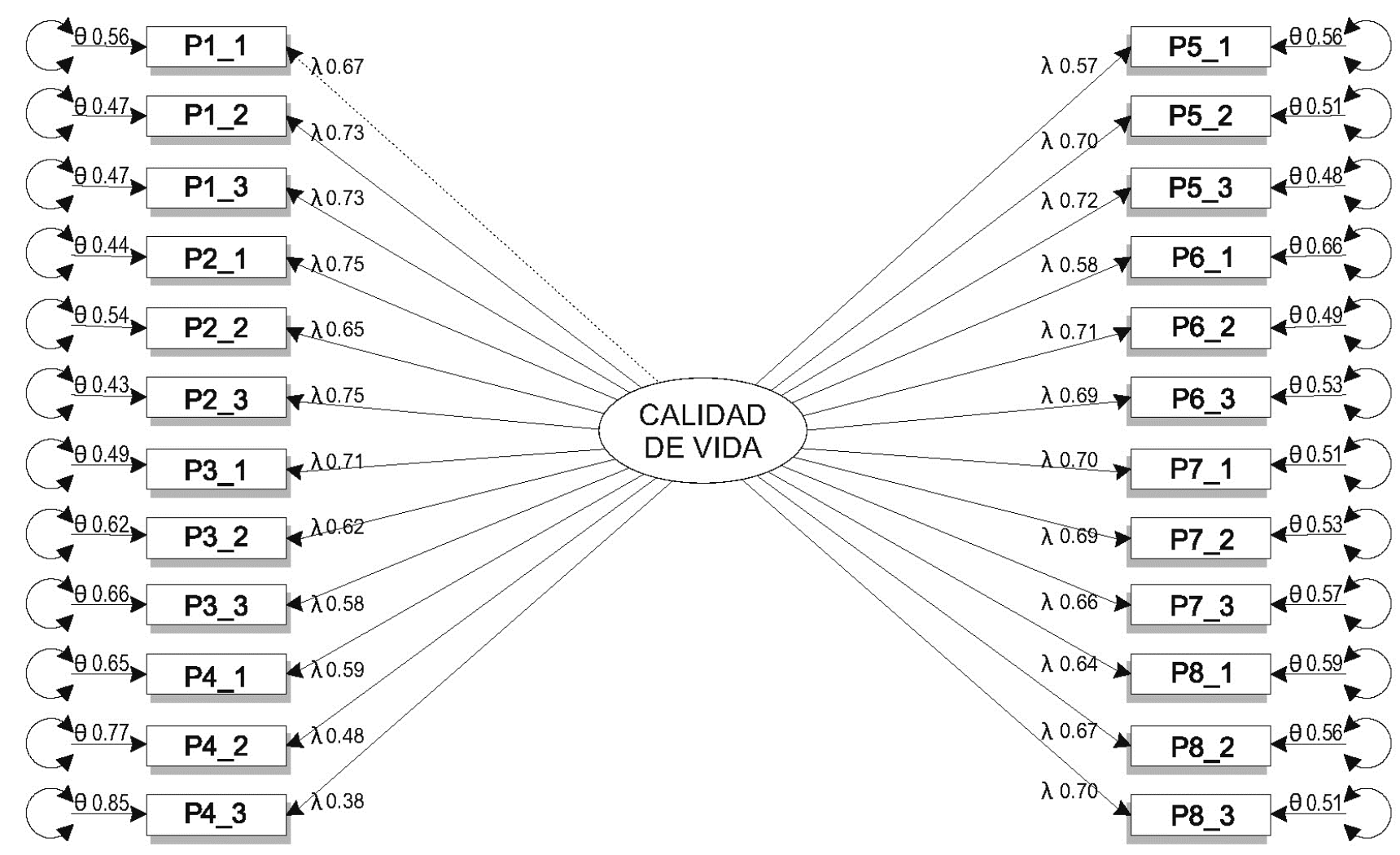

Figura 1. Parámetros estimados para el Modelo 1.

$(\theta)$ Varianza de los errores de medida (24)

$\mathrm{P}_{\mathrm{n} \_\mathrm{k}}$. Indicadores Parcels $\left(\mathrm{P}_{1,8 \_1,24}\right)$

(A) Efectos directos del factor sobre los indicadores (23) y efectos indirectos sobre los indicadores (1)

CV - Factor Calidad de Vida

$\mathrm{M}_{1}$ - Varianza de la variable latente (1)

Los resultados para el segundo modelo se observan en la Figura 2. En este caso, algunos coeficientes estimados no resultaron significativos: varianzas de los errores de medida de los indicadores P3_1 $(p=0,325)$ y P4_1 $(p=0,501)$. Los errores de predicción oscilaron entre 0,10 $\left(\mathrm{P} 4 \_1\right)$ y 0,60 (P4_3). Las cargas factoriales oscilaron entre 0,63 (P4_3) y 0,95 (P4_1), siendo todas ellas superiores a 0,50 . En cuanto a los coeficientes de correlación múltiple $\left(R^{2}\right)$, oscilaron entre 0,34 y 0,81 , de los cuales 22 superaron el $50 \%$ de la proporción de varianza. Estos resultados indicaron que la solución multifactorial del Modelo 2 mostró un ajuste aceptable comparado con la solución unifactorial del Modelo 1. El ajuste aceptable del Modelo 2 se refleja en el efecto indirecto de cada una de las dimensiones en su respectivo parcels 1 , situación que indica el efecto moderador de una tercera variable, externa al modelo. 


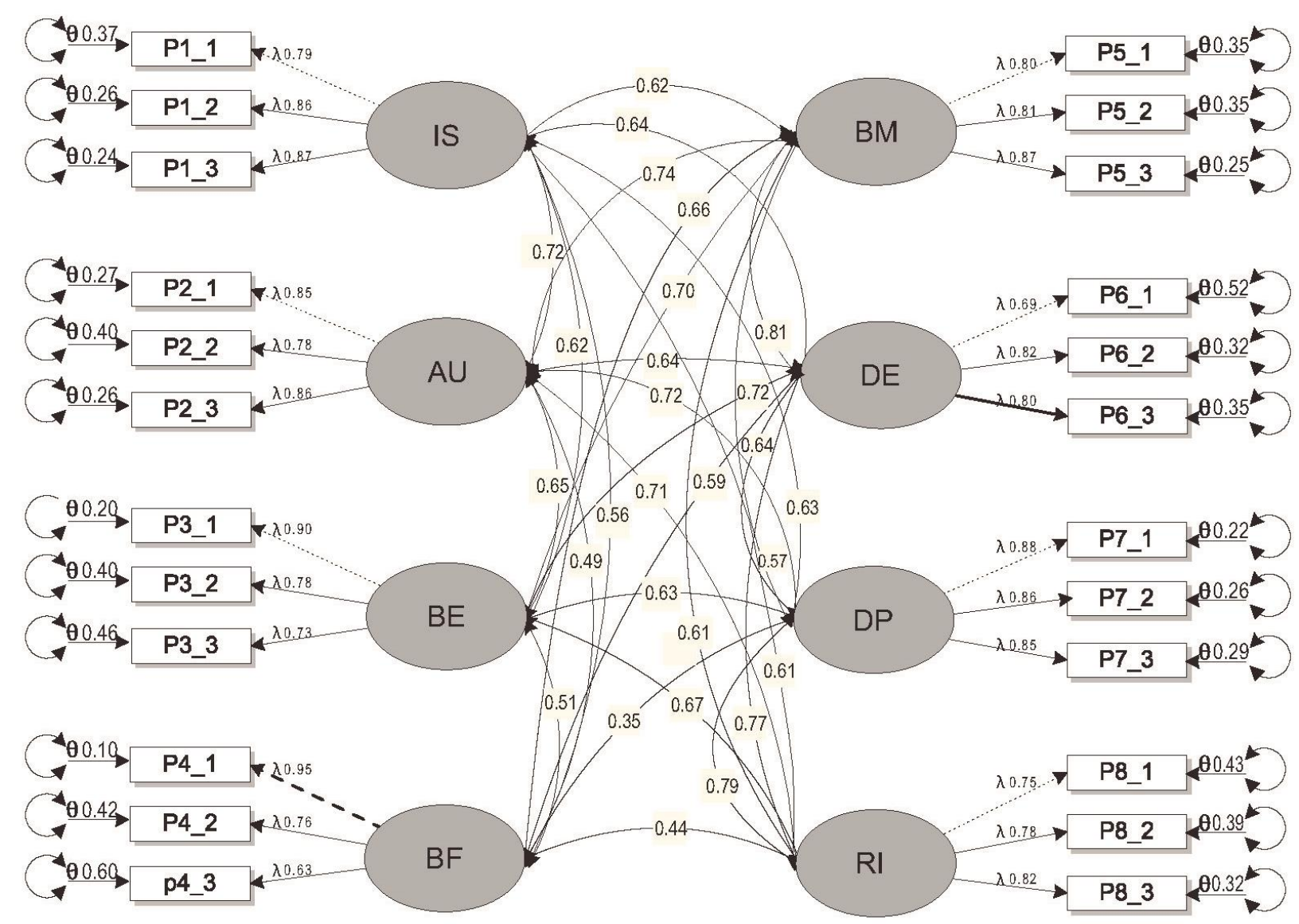

Figura 2. Parámetros estimados para el Modelo 2.

( $\theta$ ) Varianzas de los errores de medida (24)

$\mathrm{P}_{\mathrm{n} \_\mathrm{k}}$ - Indicadores Parcels $\left(\mathrm{P}_{1,8 \_1,24}\right)$

(A) Efectos directos del factor sobre los indicadores (16) y efectos indirectos sobre los indicadores (8)

Factor CV: IS - Inclusión Social; AU - Autodeterminación; BE - Bienestar Emocional; BF -

Bienestar Físico; BM - Bienestar Material; DE - Derechos; DP - Desarrollo Personal y RI -

Relaciones Interpersonales

(Ф) Covarianzas entre factores (28)

$\mathrm{M}_{2}$ - Varianzas de las variables latentes (8)

Los resultados del Modelo 3 aparecen en la Figura 3. Algunos coeficientes del modelo no fueron significativos: P3_1 $(p=0,324)$ y P4_1 $(p=0,529)$. Se observó que los errores de predicción fluctuaron entre 0,09 (P4_1) y 0,62 (P4_3). En cuanto a las saturaciones factoriales de primer orden, estas oscilaron entre 0,62 (P4_3) y 0,95 (P4_1) y las de segundo orden oscilaron entre 0,61 (Bienestar Físico) y 0,85 (Autodeterminación, Derechos y Relaciones Interpersonales). Todos los indicadores de primer orden registraron una carga factorial superior a 0,5 y solo dos indicadores fueron inferiores al $50 \%$ de la proporción de varianza. En cuanto a los indicadores de segundo orden, todas las cargas factoriales fueron superiores a 0,5 ; sin embargo, solo un indicador registró un coeficiente de correlación múltiple $\left(R^{2}\right)$ inferior a 0,5 (Bienestar Físico). Estos resultados indicaron que la solución con factores de primer y segundo orden era relativamente "aceptable" para el contexto de los datos colombianos, dado que solo se observó un ajuste de los factores de primer orden. Nótese que la variable exógena "CGCV" ejerció una influencia indirecta sobre la dimensión IS y que el efecto de cada una de las variables endógenas sobre el parcels 1 , fue indirecto, lo que muestra que las respuestas estuvieron afectadas por variables externas (moderadoras). 


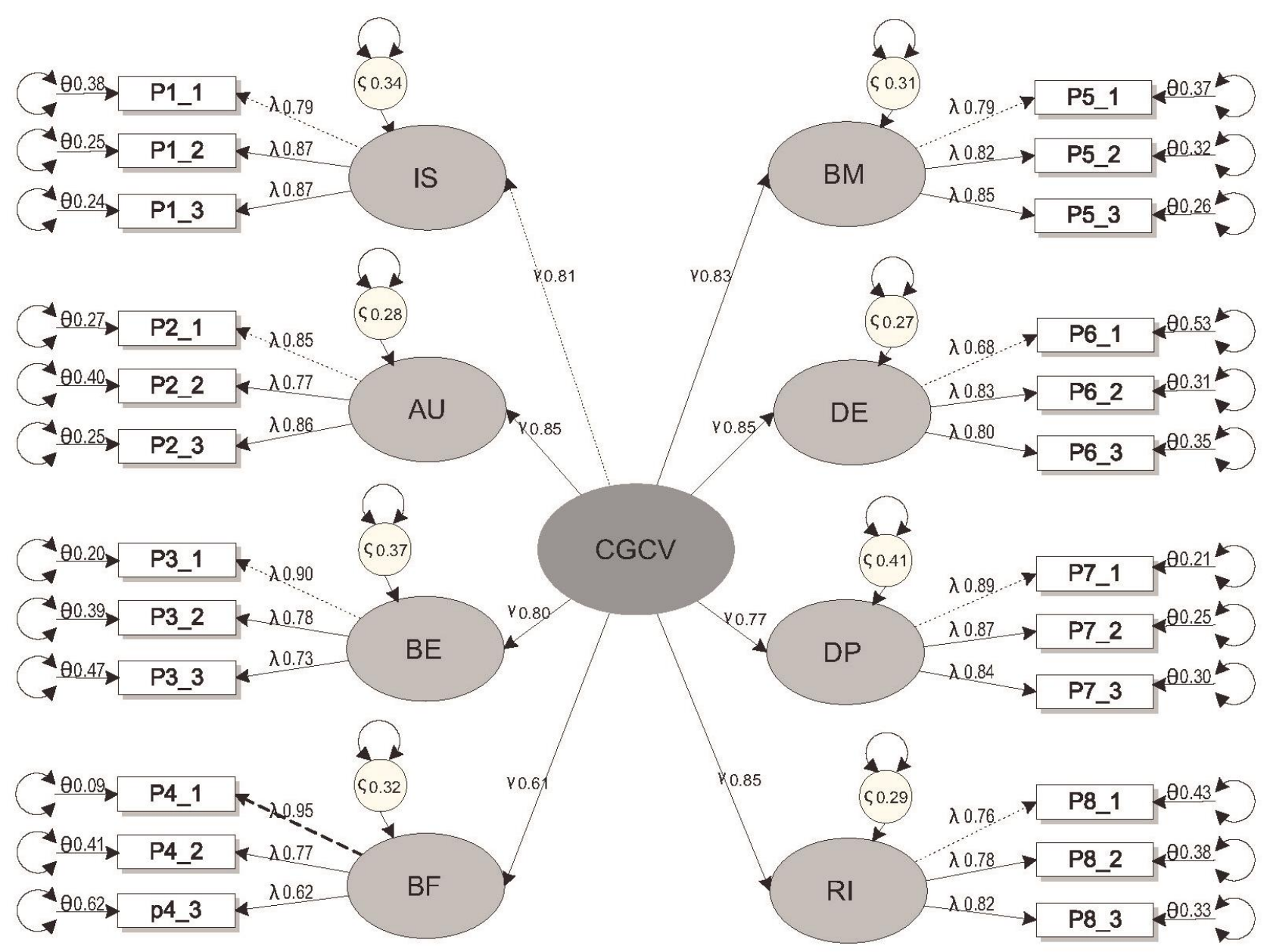

Figura 3. Parámetros estimados para el Modelo 3.

$(\theta)$ Varianzas de los errores de medida (24)

$\mathrm{P}_{\mathrm{n} \_\mathrm{k}}$ - Indicadores Parcels $\left(\mathrm{P}_{1,8 \_1,24}\right)$

(A) Efectos directos de las variables endógenas sobre los indicadores (16) y efectos indirectos de las variables de las variables endógenas sobre los indicadores (8)

Factor CV: IS - Inclusión Social; AU - Autodeterminación; BE - Bienestar Emocional; BF - Bienestar

Físico; BM - Bienestar Material; DE - Derechos; DP - Desarrollo Personal y RI - Relaciones

Interpersonales

( $\theta$ ) Errores de medida de las variables endógenas (8)

(ג) Efectos directos de la variable exógena sobre las endógenas (7) y efecto indirecto de la variable exógena sobre la endógena (1)

CGCV - Constructo General de Calidad de Vida (Factor de Segundo Orden)

(Ф) Covarianzas entre factores (28)

$\mathrm{M}_{3}$ - Varianzas de las variables latentes (1)

La solución para el Modelo 4 arrojó una matriz singular que no define positivamente los datos, en razón a la alta correlación entre algunos factores. La Tabla 2 muestra la matriz de correlaciones múltiples, observándose que oscilaron entre 0,448 (BE con BF) y 1,015 (EPW con BP). Un total de cuatro correlaciones fueron superiores a 0,90, todas ellas relacionadas con los factores de segundo orden; por esta razón, el Modelo 4 no se ajustó a los datos de las tres ciudades colombianas, aunque estuviera teóricamente definido. Estadísticamente, un valor superior a uno (1), implica un error en la estimación de los parámetros y, por tanto, en la definición de los factores de segundo orden. No se 
descarta que la falta de normalidad inicial en la distribución de los datos sea la explicación. Esto será analizado en el apartado correspondiente.

Tabla 2

Matriz de Correlaciones entre los Factores del Modelo 4

\begin{tabular}{lccccccccccc}
\hline FAC & IS & AU & BE & BF & BM & DE & DP & RI & BP & EPW & BFM \\
\hline IS & 1,000 & & & & & & & & & & \\
AU & 0,686 & 1,000 & & & & & & & & & \\
BE & 0,637 & 0,701 & 1,000 & & & & & & & & \\
BF & 0,519 & 0,482 & 0,448 & 1,000 & & & & & & & \\
BM & 0,704 & 0,654 & 0,608 & 0,663 & 1,000 & & & & & & \\
DE & 0,643 & 0,729 & 0,677 & 0,551 & 0,748 & 1,000 & & & & & \\
DP & 0,623 & 0,686 & 0,638 & 0,438 & 0,595 & 0,662 & 1,000 & & & & \\
RI & 0,684 & 0,753 & 0,699 & 0,481 & 0,652 & 0,727 & 0,684 & 1,000 & & & \\
BP & 0,789 & 0,869 & 0,807 & 0,555 & 0,753 & 0,839 & 0,790 & 0,866 & 1,000 & & \\
EPW & 0,778 & 0,882 & 0,819 & 0,667 & $\mathbf{0 , 9 0 6}$ & 0,826 & 0,802 & 0,879 & $\mathbf{1 , 0 1 5}$ & 1,000 & \\
BFM & 0,742 & 0,690 & 0,641 & 0,699 & $\mathbf{0 , 9 4 9}$ & 0,789 & 0,627 & 0,688 & 0,794 & $\mathbf{0 , 9 5 5}$ & 1,000 \\
\hline
\end{tabular}

Nota. Factores de Primer Orden: IS Inclusión Social; AU Autodeterminación; BE Bienestar Emocional; BF Bienestar Físico; BM Bienestar Material; DE Derechos; DP Desarrollo Personal; RI Relaciones Interpersonales.

Factores de Segundo Orden: BP Bienestar Personal; EPW Empoderamiento y BFM Bienestar Físico y Material.

Los valores ennegrecidos corresponden a las correlaciones más altas $(\geq 0,90)$, entre los factores de segundo orden.

Los resultados del Modelo 5 se muestran en la Figura 4. Los coeficientes de las varianzas de los indicadores P3_1 y P4_1 no fueron significativos. Los errores de predicción estuvieron alrededor de 0,09 (P4_1) y 0,62 (P4_3). Las saturaciones factoriales de primer orden oscilaron entre 0,62 (P4_3) y 0,96 (P4_1) y los 24 indicadores tuvieron una carga factorial por encima de 0,5. En cuanto a las cargas del segundo orden, los valores oscilaron entre 0,65 y 0,89 . Se deduce que los coeficientes de correlación múltiple $\left(R^{2}\right)$ para el primer orden se encuentran entre 0,38 y 0,92, es decir, la mayoría superó el $50 \%$ de la proporción de varianza de las variables observadas, que logra ser explicada por el único factor latente, a excepción del indicador P4_3. Se encuentra que las correlaciones entre las variables exógenas (de segundo orden) superan el valor de 0,90, lo que trae problemas para la identificación empírica del modelo, posiblemente asociado a la multicolinealidad inicial de las variables. Además, se observa que el efecto de cada una de las dimensiones sobre el parcels 1 , es indirecto.

Paralelamente, se identifica el efecto indirecto de los factores de segundo orden (variables exógenas), sobre algunas de las dimensiones de primer orden, lo que reafirma influencia de variables externas que provocan escaso ajuste de los datos al modelo 5.

Luego de estimar los parámetros, se evaluó la adecuación de los modelos teóricos al conjunto de datos del estudio. La Tabla 3 muestra algunos de los índices de bondad de ajuste más comunes, desde las medidas absolutas de ajuste hasta las medidas incrementales y medidas de ajuste parsimonioso para la escala KidsLife.

Como se observa en la Tabla 3 , a partir del índice $\chi^{2}$ S-B, se rechaza la hipótesis de que el modelo se ajusta perfectamente a los datos de la población únicamente para el Modelo 1, dado que no resulta significativo. De acuerdo con los datos, el Modelo 2 registra una $X^{2}$ menor, pero como este es un estadístico sensible al tamaño muestral, se recurre a los índices de ajuste. 


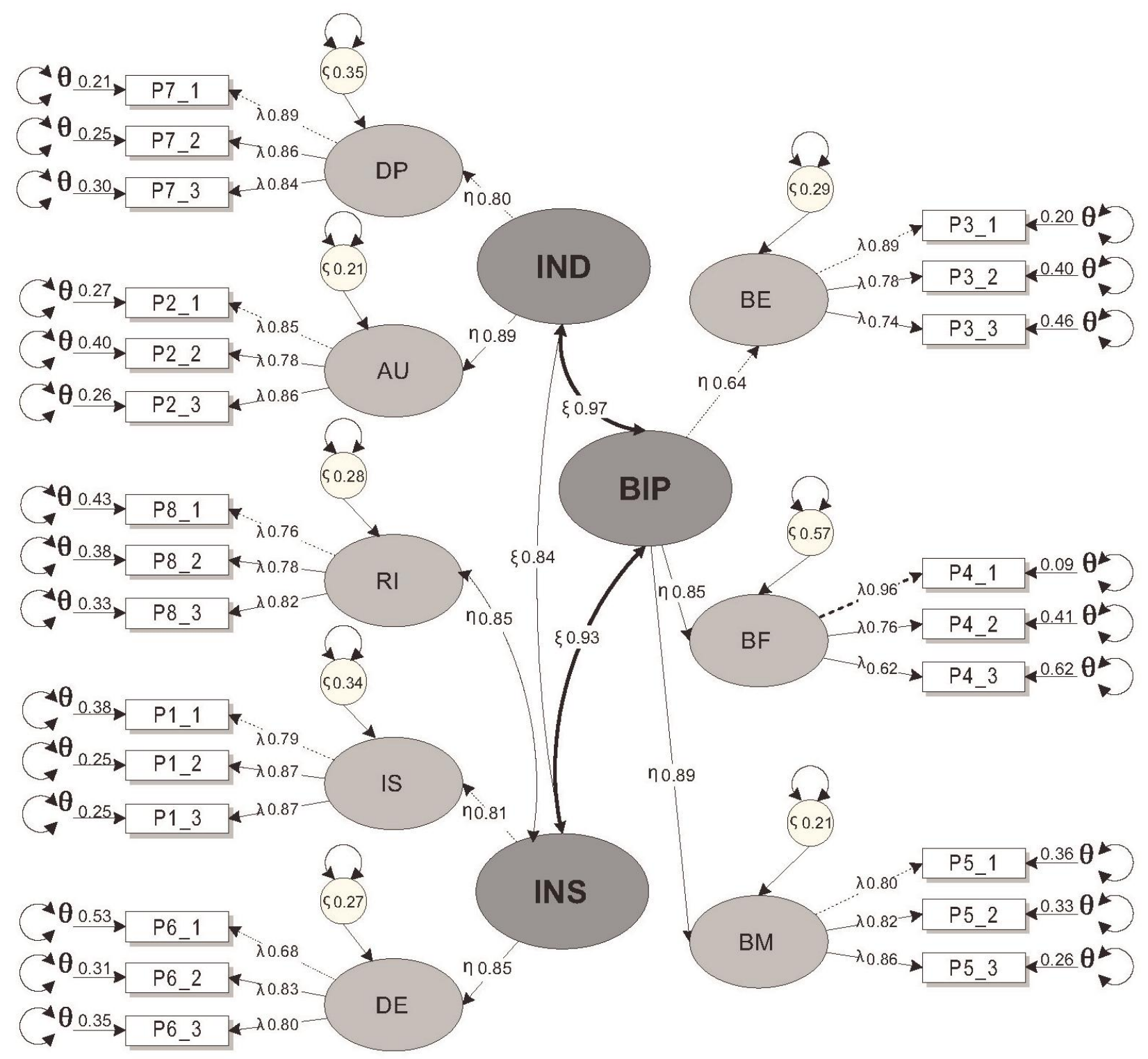

Figura 4. Parámetros estimados para el Modelo 5.

$(\theta)$ Varianzas de los errores de medida (24)

$\mathrm{P}_{\mathrm{n} \_\mathrm{k}}$ - Indicadores Parcels $\left(\mathrm{P}_{1,8 \_1,24}\right)$

(A) Efectos directos de las variables endógenas sobre los indicadores (16) y efectos indirectos de las variables de las variables endógenas sobre los indicadores (8)

Factor CV: IS - Inclusión Social; AU - Autodeterminación; BE - Bienestar Emocional; BF - Bienestar Físico; BM - Bienestar Material; DE - Derechos; DP - Desarrollo Personal y RI - Relaciones Interpersonales

( $\theta$ ) Errores de medida de las variables latentes endógenas (8)

$(\Lambda)$ Efectos directos de la variable exógena sobre las endógenas (5) y efecto indirecto de la variable exógena sobre la endógena (3)

Factores de Segundo Orden: IND - Independencia; INS - Integración Social y BIP - Bienestar Personal (Ф) Covarianzas entre factores (3)

$\mathrm{M}_{5}$ - Varianzas de las variables latentes (3) 
Tabla 3

Índices de Ajuste de los Modelos puestos a prueba para la Escala KidsLife

\begin{tabular}{|c|c|c|c|c|c|c|c|c|c|}
\hline \multirow{2}{*}{ Modelo } & \multicolumn{4}{|c|}{ Medidas absolutas de ajuste } & \multicolumn{2}{|c|}{$\begin{array}{c}\text { Medidas } \\
\text { incrementales de } \\
\text { ajuste }\end{array}$} & \multicolumn{3}{|c|}{ Medidas de ajuste parsimonioso } \\
\hline & SRMR & $\chi^{2} \mathrm{~S}-\mathrm{B}$ & $g l$ & $p$ & CFI & TLI & RMSEA & $\begin{array}{l}\text { RMSEA } 90 \% \\
\text { IC }\end{array}$ & $p$ \\
\hline Modelo 1 & 0,093 & 389,6 & 252 & $<0,001$ & 0,985 & 0,983 & 0,051 & $0,041,0,060$ & 0,447 \\
\hline Modelo 2 & 0,051 & 135,2 & 224 & 0,997 & 0,999 & 0,998 & 0,001 & $0,000,0,002$ & 0,999 \\
\hline Modelo 3 & 0,066 & 218,4 & 244 & 0,879 & 0,999 & 0,997 & 0,001 & $0,000,0,002$ & 0,999 \\
\hline Modelo 4 & 0,061 & 185,9 & 241 & 0,979 & 0,996 & 0,995 & 0,002 & $0,001,0,003$ & 0,996 \\
\hline Modelo 5 & 0,063 & 200,0 & 241 & 0,975 & 0,996 & 0,995 & 0,001 & $0,000,0,002$ & 0,999 \\
\hline
\end{tabular}

Nota. Las cifras ennegrecidas corresponden a los datos que más se acercan a los valores de referencia de los índices de bondad de ajuste: $\mathrm{SRMR}=<0,05 ; \mathrm{CFI}=>0,90 ; \mathrm{TLI}=>0,95$ y $\mathrm{RMSEA}=\leq 0,05$.

Comparativamente, el Modelo 2 (ocho dimensiones correlacionadas entre sí), captura mejor los datos, se ajusta a los valores de referencia, se acomoda en forma más precisa a los datos e informa acerca de que las variables están debidamente correlacionadas, lo que significa que ofrece una descripción más simple y sencilla de los datos. El Modelo 2 describe las correlaciones entre las variables en forma aceptable y con un mínimo error de 0,051 puntos: la diferencia de 0,001 puntos respecto al valor de $\mathrm{SRMR} \leq 0,05$ que indica un buen ajuste, es atribuible a efectos residuales mínimos en los datos.

La Tabla 4 muestra que la fiabilidad compuesta de las ocho dimensiones de CV superó el valor de 0,60 y que se ubican por encima de 0,80. La fiabilidad de la Escala KidLife, en general, resulta ser mucho más alta. En cuanto a la varianza media extractada, es superior al 50\% en las ocho dimensiones de la escala. Un análisis global sugiere que el Modelo 2 de ocho factores correlacionados, informa positivamente acerca de la validez y confiabilidad de las dimensiones de CV en la que se basa la Escala KidsLife.

Tabla 4

Fiabilidad Compuesta y Varianza Media Extractada de la Escala KidsLife

\begin{tabular}{lcc}
\hline \multicolumn{1}{c}{ Dimensión } & Fiabilidad compuesta & Varianza media extractada \\
\hline Inclusión Social & 0,879 & 0,709 \\
Autodeterminación & 0,869 & 0,690 \\
Bienestar Emocional & 0,845 & 0,647 \\
Bienestar Físico & 0,830 & 0,626 \\
Bienestar Material & 0,866 & 0,683 \\
Derechos & 0,817 & 0,600 \\
Desarrollo Personal & 0,897 & 0,743 \\
Relaciones Interpersonales & 0,828 & 0,617 \\
Total & 0,979 & 0,665 \\
\hline
\end{tabular}

La Tabla 5 muestra las correlaciones entre los constructos o dimensiones del Modelo 2 seleccionado. Se encuentran correlaciones muy satisfactorias, puesto que no superan el valor de 0,90 y tampoco se registran correlaciones muy bajas $(r<0,20)$. Las correlaciones están comprendidas entre 0,345 y 0,805 , corresponden a los parámetros estimados para el Modelo 2, que ya fueron representados en la Figura 2. 
Tabla 5

Coeficientes de Correlación entre las Variables Latentes del Modelo 2 (Matriz de Correlaciones)

\begin{tabular}{lcccccccc}
\hline \multicolumn{1}{c}{ Dimensión } & IS & AU & BE & BF & BM & DE & DP & RI \\
\hline Inclusión Social (IS) & 1,000 & & & & & & & \\
Autodeterminación (AU) & 0,724 & 1,000 & & & & & & \\
Bienestar Emocional (BE) & 0,618 & 0,648 & 1,000 & & & & & \\
Bienestar Físico (BF) & 0,555 & 0,484 & 0,506 & 1,000 & & & & \\
Bienestar Material (BM) & 0,622 & 0,735 & 0,703 & 0,664 & 1,000 & & & \\
Derechos (DE) & 0,642 & 0,639 & 0,717 & 0,589 & 0,805 & 1,000 & & \\
Desarrollo Personal (DP) & 0,629 & 0,716 & 0,628 & 0,345 & 0,565 & 0,639 & 1,000 & \\
Relaciones Interpersonales (RI) & 0,706 & 0,714 & 0,666 & 0,444 & 0,611 & 0,764 & 0,792 & 1,000 \\
\hline
\end{tabular}

Posteriormente, se examinó la consistencia interna de la Escala KidsLife, registrando un alfa de Cronbach de 0,964 para la escala total que corresponde a un muy buen nivel. La Tabla 6 reúne los valores encontrados para las ocho dimensiones de la escala. Como era de esperarse, los valores son muy similares a los de la fiabilidad compuesta; por esta razón, se ratifica la consistencia interna de la Escala KidsLife con base en el modelo de ocho factores de CV.

Tabla 6

Alfa de Cronbach para el Modelo 2 de Ocho Dimensiones Correlacionadas

\begin{tabular}{lc}
\hline \multicolumn{1}{c}{ Dimensión } & Alfa de Cronbach \\
\hline Inclusión Social & 0,869 \\
Autodeterminación & 0,876 \\
Bienestar Emocional & 0,835 \\
Bienestar Físico & 0,805 \\
Bienestar Material & 0,823 \\
Derechos & 0,793 \\
Desarrollo Personal & 0,889 \\
Relaciones Interpersonales & 0,835 \\
Total & 0,964 \\
\hline
\end{tabular}

En resumen, se corroboró que el Modelo 2 (ocho factores correlacionados entre sí), se ajustaba aceptablemente a los datos recogidos en el contexto de tres ciudades colombianas; además, se confirmó la fiabilidad, validez y adecuada consistencia interna de la Escala KidsLife, diseñada a partir del mismo. Se corroboró la multidimensionalidad del concepto CV, dado que el Modelo 2 se ajustó de manera simple a los datos, describiéndolos en forma completa. Los Modelos 3, 4 y 5 no lograron un ajuste completo.

Por último, cabe analizar el comportamiento del parcel P4_3 de la dimensión BF dado que aportó la menor saturación factorial $(0,63)$ en los distintos modelos; en este caso, las respuestas al ítem 1 (consumo balanceado de alimentos), se realizó en función de la capacidad económica de la familia y no del bienestar físico, aunque se reconocía su importancia para la salud; esto muestra la necesidad de analizar la influencia de las variables externas (aspectos socioeconómicos y culturales) en las respuestas a la Escala.

\section{Discusión}

Se ratifica que la estructura interna de la Escala KidsLife se basa en un modelo compuesto por ocho factores correlacionados entre sí: Inclusión Social, Autodeterminación, Bienestar Emocional, Bienestar Físico, Bienestar Material, Derechos, Desarrollo Personal y Relaciones Interpersonales, propuesto por Schalock y Verdugo (2007). Se corrobora la multidimensionalidad del constructo CV y se encuentra 
evidencia de su validez para la aplicación en la práctica profesional en el campo de la discapacidad intelectual.

Se reconoce la utilidad del modelo teórico Schalock y Verdugo para el estudio de la CV, hallazgo que concuerda con lo reportado por estudios realizados en Portugal, Brasil y España, en los que también se reportó el ajuste de la estructura factorial del modelo (8 factores de primer orden correlacionados entre sí) a los datos obtenidos por las investigaciones (Bredemeier et al., 2014; Gómez, Alcedo, Arias et al., 2016; Guillén et al., 2015; Simões et al., 2016).

Aunque se verificó el ajuste aceptable de los datos obtenidos con la Escala KidsLife a la propuesta de Schalock y Verdugo (2007), el análisis de los datos de las tres ciudades colombianas no permitió identificar factores de segundo orden; los índices de ajuste ratificaron la multidimensionalidad e interdependencia del constructo CV, en la que todos los factores fueron igualmente determinantes. Se plantea la pertinencia de profundizar en la composición de los factores de segundo orden, explicando sus diferencias de acuerdo con cada contexto investigado.

Es indispensable notar que los factores de segundo orden corresponden a un segundo nivel de explicación acerca de las dimensiones constitutivas de la CV, que obedecen a un orden jerárquico que es, a su vez, relativo a las condiciones económicas y socioculturales concretas de las que se extraen los datos. La existencia de una estructura jerárquica no corresponde a un ordenamiento lineal, sino interactivo, lo que explica que las dimensiones propuestas por el Modelo 2 de Schalock y Verdugo (2007), sean igualmente importantes para la evaluación de la CV en el contexto actual de las tres ciudades de Colombia.

Respecto a las dificultades para el ajuste de los demás modelos a los datos colombianos, se anota que el "posible" único factor de segundo orden identificado en el Modelo 3, fue la: "Capacidad de Ser Autónomo en una Sociedad de Derechos", diferente al "Constructo General de CV", único factor de segundo orden reportado por Wang et al. (2010). Por lo tanto, este modelo no logró el ajuste debido. Sin embargo, los datos sugieren que el factor diferencial de segundo orden podría ser la "Capacidad de Ser Autónomo en una Sociedad de Derechos", aunque se tendrían que realizar nuevos estudios con una población similar para determinarlo. Igualmente, se concluye que no es posible la generalización de factores de segundo orden, pues estos son relativos al análisis correlacional de los datos de primer orden provenientes de la población analizada.

Es necesario tener en cuenta que el propósito de Wang fue analizar datos provenientes de 14 países, con el fin de establecer la estructura jerárquica de las dimensiones del constructo CV para la identificación de factores de segundo orden, recurriendo a una analogía con el factor g de la Escala de Weschler de Inteligencia; no obstante, tal analogía no es pertinente porque se trata de dos conceptos e instrumentos de medición que pertenecen a dominios evaluativos diferentes. Además, no se comparte la pretensión de "universalizar" la composición de los factores de segundo orden entre diferentes países o culturas, pues se trata de un objetivo contrario a la conceptualización de interculturalidad y estudios interculturales.

La construcción de una teoría de CV y el desarrollo de modelos explicativos sobre ella requiere tener en cuenta que los factores de segundo orden son útiles para analizar las diferencias entre los países y las culturas, dado que se está hablando de condiciones de vida contextualizadas. En este sentido, los factores de segundo orden corresponden a explicaciones que amplían la comprensión de los datos iniciales, de forma que ayudan a entender las metas para alcanzar un desarrollo social equitativo en cada país, a partir de la identificación de las necesidades de cada contexto.

Por otra parte, el registro de correlaciones superiores a 0,90 en los factores de segundo orden que no permitieron la identificación del Modelo 4, coincide con lo reportado por Gómez et al., (2011), quienes también encontraron correlaciones superiores, incluyendo $r=1,015$ entre EPW y BP; estos valores indican que la diferenciación de los factores de segundo orden no es clara y se presume que se deriva de su definición ligada al bienestar material (disponibilidad de ayudas técnicas y tecnológicas adaptadas). Los resultados ratifican la necesidad de profundizar en la definición de los factores de segundo orden, reconociendo los efectos moderadores de variables externas al modelo (características del contexto socioeconómico y diferencias culturales de cada país).

En lo que se refiere al Modelo 5, las correlaciones altas entre las variables de segundo orden (exógenas) afectaron negativamente el ajuste de los datos, resultado que se interpretaría como producto de la multicolinealidad entre ellas. Sin embargo, el análisis muestra que los factores de segundo orden no saturaron adecuadamente las dimensiones IS, BE y DP, debido a la influencia de las variables externas. Es 
interesante notar que el Modelo 5 propone factores de segundo orden que reflejan el carácter interdependiente del concepto CV (individuo, interacción y sociedad).

En el plano metodológico, el tamaño de la muestra constituyó una limitante, por cuanto pudo afectar la significatividad estadística de los índices de ajuste para los modelos. Sería necesario ampliar la muestra, de forma que se asegure una distribución normalizada, se incluya un número equivalente de hombres y mujeres, y un número similar de personas residentes en las diferentes zonas de estratificación socioeconómica reconocidas en Colombia, precisamente porque se trata de condiciones muy heterogéneas.

Finalmente, se propone incorporar posibles variables moderadoras externas al análisis de CV; por ejemplo, variables del informante (rol que asume en la vida de la persona discapacitada) o variables de la institución de apoyo y el tipo de programa educativo que ofrece y variables relacionadas con las condiciones socioeconómicas de la familia (Córdoba, Salamanca, \& Mora, 2019). Se recomienda emplear métodos no paramétricos y muestreos por conveniencia que faciliten el análisis contextualizado de la información sobre $\mathrm{CV}$, en términos de la localización y caracterización de la CV de la población con discapacidad, teniendo en cuenta la dificultad de lograr un muestreo aleatorio.

\section{Referencias}

American Educational Research Association, American Psychological Association \& National Council on Measurement in Education (2014). Standards for educational and psychological testing ( $8^{\mathrm{a}}$ ed.). Washington, DC: American Educational Research Association.

Arenas, B., Jaramillo, J., Marmolejo, P. \& Cruz, C. (2013). Calidad de vida e inclusión laboral de personas con discapacidad. Cali, 2008-2011. Ciencia \& Salud, $\quad$ 1(3), $\quad 39-47 . \quad$ Extraído de https://repository.usc.edu.co/bitstream/20.500.12421/820/1/5\%20Calidad\%20de\%20vida\%20e\%20inclusi\%C3\%B3n\%20labor al\%20de\%20personas\%20con\%20discapacidad.\%20Cali\%2C\%202008-2011.pdf

Arias Martínez, B. (2008). Desarrollo de un ejemplo de análisis factorial confirmatorio con LISREL, AMOS y SAS. En M. A. Verdugo, M. Crespo, M. Badía \& B. Arias (Coords.), Metodología en la investigación sobre discapacidad. Introducción al uso de las ecuaciones estructurales (pp. 75-120). Salamanca, España: Universidad de Salamanca, Instituto Universitario de Integración en la Comunidad.

Arribas, A. (2006). Adaptación transcultural de instrumentos. Guía para el proceso de validación de instrumentos de tipo encuestas. Revista Científica de la Asociación Médica de Bahía Blanca, 16, 74-82. Extraído de https://pesquisa.bvsalud.org/portal/resource/pt/biblio-1007566

Asociación Médica Mundial (2017). Declaración de Helsinki de la AMM - principios éticos para las investigaciones médicas en seres humanos. Ferney-Voltaire, Francia: Autor. Extraído de https://www.wma.net/es/policies-post/declaracion-de-helsinki-de-la-ammprincipios-eticos-para-las-investigaciones-medicas-en-seres-humanos/

Bangdiwala, S. I. \& Bryan, H. E. (1987). Using SAS software graphical procedures for the observer agreement chart, en SAS Institute, SUGI 12: Proceedings of the Twelfth Annual SAS Users Group International Conference (pp. 1083-1088). Cary, NC: SAS Institute.

Bentler, P. M. \& Chu, C -P. (1987). Practical issues in structural modeling. Sociological Methods \& Research, 16, 78-117. https://doi.org/10.1177/0049124187016001004

Breckler, S. J. (1990). Applications of covariance structure modeling in psychology: cause for concern?. Psychological Bulletin, 107, 260-273. https://doi.org/10.1037/0033-2909.107.2.260

Bredemeier, J., Peretti Wagner, G., Agranonik, M., Spalding Perez, T. \& Fleck, M. P. (2014). The World Health Organization Quality of Life instrument for people with intellectual and physical disabilities (WHOQOL-Dis): Evidence of validity of the Brazilian version. BMC Public Health, 14, artículo 538. https://doi.org/10.1186/1471-2458-14-538

Brown, T. A. (2006). Confirmatory factor analysis for applied research. New York: Guilford Press.

Carretero-Dios, H. \& Pérez, C. (2007). Standards for the development and review of instrumental studies: Considerations about test selection in psychological research. International Journal of Clinical and Health Psychology, 7, 863-882. Extraído de https://www.redalyc.org/pdf/337/33770319.pdf

Colombia, DANE (2018). Estratificación socioeconómica - preguntas frecuentes. Bogotá, Colombia: Departamento Administrativo Nacional de Estadística. Extraído de http://www.dane.gov.co/index.php/116-espanol/informacion-georreferenciada/2421estratificacion-socioeconomica-preguntas-frecuentes

Colombia, Ministerio de Salud (1993). Resolución 8430 de 1993 por la cual se establecen las normas científicas, técnicas y administrativas para la investigación en salud. Bogotá, Colombia: Autor.

Colombia, Ministerio de Salud y Protección Social (2013). Resolución 1441 de 2013 por la cual se definen los procedimientos y condiciones que deben cumplir los prestadores de servicios de salud para habilitar los servicios y se dictan otras disposiciones. Bogotá, Colombia: Autor.

Congreso de Colombia (Ministerio de Educación Nacional, Ministerio de Trabajo y Seguridad Social, Ministerio de Salud \& Ministerio de Transporte) (1997). Ley 361 de 1997 por la cual se establecen los mecanismos de integración social de las personas con limitación y se dictan otras disposiciones. Bogotá, Colombia: Diario Oficial 42.978.

Córdoba Andrade, L., Henao Lema, C. P. \& Verdugo Alonso, M. Á. (2016). Calidad de vida de adultos colombianos con discapacidad intelectual. Revista Hacia la Promoción de la Salud, 21(1), 91-105. https://doi.org/10.17151/hpsal.2016.21.1.8

Córdoba, L., Mora, A., Bedoya, Á. \& Verdugo, M. A. (2007). Familias de adultos con discapacidad intelectual en Cali, Colombia, desde el modelo de calidad de vida. Psykhe, 16(2), 29-42. https://doi.org/10.4067/S0718-222820070002000256

Córdoba-Andrade. L., Salamanca Duque, L. M. \& Mora Anto, A. (2018). Versión de la Escala KidsLife para tres ciudades de Colombia. Ponencia presentada en las X Jornadas Científicas Internacionales de Investigación sobre Personas con Discapacidad. Salamanca, España: Universidad de Salamanca. Libro de Actas en CD, p. 1-10. 
Córdoba-Andrade, L., Mora Anto, A. \& Salamanca Duque, L. M. (2019). Versión para Colombia de la Escala KidsLife para la evaluación de la calidad de vida en personas de 4 a 21 años con discapacidad intelectual o múltiple. Ibagué, Colombia: Sello Editorial Universidad del Tolima.

Córdoba, L., Salamanca, L., M., \& Mora, A. (2019). Calidad de Vida en Personas con Discapacidad Intelectual y Múltiple De 4 a 21 Años de Edad. Psychologia, 13(2), 79-93. https://doi.org/10.21500/19002386.4014.

Cupani, M. (2012). Análisis de ecuaciones estructurales: conceptos, etapas de desarrollo y un ejemplo de aplicación. Revista Tesis, 1, 186-199. Extraído de https://revistas.unc.edu.ar/index.php/tesis/article/view/2884

George, D. \& Mallery, P. (2001). SPSS for Windows step by step: A simple guide and reference. Boston, MA: Allyn \& Bacon.

Gómez, L. E., Alcedo, M. Á., Arias, B., Fontanil, Y., Arias, V. B., Monsalve, A. \& Verdugo, M. Á. (2016). A new scale for the measurement of quality of life in children with intellectual disability. Research in Developmental Disabilities, 53-54, 399-410. https://doi.org/10.1016/j.ridd.2016.03.005

Gómez, L. E., Alcedo, M. A., Verdugo, M. A., Arias, B., Fontanil, Y., Arias, V. B. ... Morán, L. (2016). Escala KidsLife: evaluación de la calidad de vida de niños y adolescentes con discapacidad intelectual (Colecciones Herramientas 10). Salamanca, España: Universidad de Salamanca, Instituto Universitario de Integración en la Comunidad.

Gómez, L. E., Peña, E., Alcedo, M. A., Monsalve, A., Fontanil, Y., Verdugo, M. A. \& Arias, V. (2014). El Constructo de Calidad de Vida en niños y adolescentes con Discapacidades Múltiples y Profundas: Propuesta para su Evaluación. Siglo Cero, 45, 1, 249 , 56-69. Extraído de https://sid.usal.es/idocs/F8/ART20387/constructo_calidad_vida_249.pdf

Gómez, L. E., Verdugo, M. A. \& Arias, B. (2015). Validity and reliability of the INICO-FEAPS Scale: An assessment of quality of life for people with intellectual and developmental disabilities. Research in Developmental Disabilities, 36, 600-610. https://doi.org/10.1016/j.ridd.2014.10.049

Gómez, L. E., Verdugo, M. Á., Arias, B. \& Arias, V. (2011). A comparison of alternative models of individual quality of life for social service recipients. Social Indicators Research, 101, 109-126. https://doi.org/10.1007/s11205-010-9639-y

Gómez, L. E., Verdugo, M. Á., Arias, B., Navas, P. \& Schalock, R. L. (2013). The development and use of provider profiles at the organization and systems level. Evaluation and Program Planning, 40, 17-26. https://doi.org/10.1016/j.evalprogplan.2013.05.001

Guillén, V. M., Verdugo, M. Á., Arias, B. \& Vicente, E. (2015). Development of a support needs assessment scale for children and adolescents with intellectual disabilities. Anales de Psicología, 31, 137-144. https://doi.org/10.6018/analesps.31.1.166491

Hair Jr., J. F., Black, W. C., Babin, B. J., Anderson, R. E. \& Tatham, R. L. (2006). Multivariate data analysis (6a ed.). Upper Saddle River, NJ: Pearson Education.

Holt, J. K. (2004, October). Item parceling in structural equation models for optimum solutions. Ponencia presentada en la 2004 Annual Meeting of the Mid-Western Educational Research Association, Columbus, OH, Estados Unidos.

Hu, L. \& Bentler, P. M. (1999). Cutoff criteria for fit indexes in covariance structure analysis: Conventional criteria versus new alternatives. Structural Equation Modeling, 6, 1-55. https://doi.org/10.1080/10705519909540118

Isaacs, B., Wang, M., Samuel, P., Ajuwon, P., Baum, N., Edwards, M. \& Rillotta, F. (2012). Testing the factor structure of the Family Quality of Life Survey - 2006. Journal of Intellectual Disability Research, 56, 17-29. https://doi.org/10.1111/j.13652788.2011.01392.x

Jenaro, C., Verdugo, M. A., Caballo, C., Balboni, G., Lachapelle, Y., Otrebski, W. \& Schalock, R. L. (2005). Cross-cultural study of person-centred quality of life domains and indicators: A replication. Journal of Intellectual Disability Research, 49, 734-739. https://doi.org/10.1111/j.1365-2788.2005.00742.x

Kline, R. B. (2005). Principles and practice of structural equation modeling (2a ed.). Ney York, NY: Guilford Press.

Lomax, R. G. (1982). A guide to LISREL-type structural equation modeling. Behavior Research Methods \& Instrumentation, $14,1-8$. https://doi.org/10.3758/BF03202105

Muñiz, J., Elosua, P. \& Hambleton, R. K. (2013). Directrices para la traducción y adaptación de los tests: segunda edición. Psicothema, 25, 151-157. https://doi.org/10.7334/psicothema2013.24

Naciones Unidas (2006). Convención de las Naciones Unidas sobre los Derechos de las Personas con Discapacidad. Naciones Unidas, Personas con Discapacidad: Departamento de Asuntos Económicos y Sociales. Extraído de https://www.un.org/development/desa/disabilities-es/convencion-sobre-los-derechos-de-las-personas-con-discapacidad-2.html

Padilla Muñoz, A. (2011). Inclusión educativa de personas con discapacidad. Revista Colombiana de Psiquiatría, 40, 670-699. Extraído de http://www.scielo.org.co/pdf/rcp/v40n4/v40n4a07.pdf

R Core Team (2016). R: A language and environment for statistical computing. Wien, Austria: R Foundation for Statistical Computing. Extraído de http://www.R-project.org/

Salamanca-Duque, L.M., Mora-Anto, A. \& Córdoba-Andrade, L. (2019). Adaptación Transcultural para Colombia de la Escala Kidslife para personas con Discapacidad Intelectual y Múltiple. Revista de la Facultad de Medicina de la Universidad Nacional, 67(2), 285-292. https://doi.org/10.15446/revfacmed.v67n2.68219

Schalock, R. L., Verdugo, M. A., Jenaro, C., Wang, M., Wehmeyer, M., Xu, J., \& Lachapelle, Y. (2005). Cross-cultural study of quality of life indicators. American Journal on Mental Retardation, 110, 298-311.

Schalock, R. L. \& Verdugo Alonso, M. A. (2007). El concepto de calidad de vida en los servicios y apoyos para personas con discapacidad intelectual. Siglo Cero: Revista Española sobre Discapacidad Intelectual, 38(224), 21-36. Extraído de https://www.plenainclusion.org/sites/default/files/224_articulos2.pdf

Simões, C., Santos, S. \& Biscaia, R. (2016). Validation of the Portuguese version of the Personal Outcomes Scale. International Journal of Clinical and Health Psychology, 16, 186-200. https://doi.org/10.1016/j.ijchp.2015.11.002

SISBEN (2013, abril). Sistema de Identificación de Potenciales Beneficiarios de Programas Sociales en Colombia. Bogotá D. C., Departamento Nacional de Planeación. Extraído de https://anda.dnp.gov.co/index.php/catalog/94/study-description

Temperaal, D. T., Schim, D, S. \& Gijselaers, W. H. (2007). A structural equation model analyzing the relationship of students' attitudes toward statistics, prior reasoning abilities and course performance. Statistics Education Research Journal, 6(2), 78-102. Extraído de https://pdfs.semanticscholar.org/3c3f/cec412a781c87ab6c923deceaa6c456ade55.pdf

Verdugo, M. Á., Gómez, L. E., Arias, B. \& Navas, P. (2012). Evidencias de validez del modelo de calidad de vida de ocho dimensiones y aplicación de la Escala Integral en distintos países. En M. A. Verdugo Alonso, R. Canal Bedia, C. Jenaro Río, M. Badia Corbella \& A. -L. Aguado Díaz (Eds.), Aplicación del paradigma de calidad de vida a la intervención con personas con discapacidad desde una perspectiva integral. Investigaciones desarrolladas por el Grupo de Investigación de Excelencia de la Junta de Castilla y León 
"GR197" (2009-2011) (Colección Investigación 7/2012; pp. 11-26). Salamanca, España: Universidad de Salamanca, Instituto Universitario de Integración en la Comunidad.

Verdugo, M. A., Navas, P., Gómez, L. E. \& Schalock, R. L. (2012). The concept of quality of life and its role in enhancing human rights in the field of intellectual disability. Journal of Intellectual Disability Research, 56, 1036-1045. https://doi.org/10.1111/j.13652788.2012.01585.x

Wang, M., Schalock, R. L., Verdugo, M. A. \& Jenaro, C. (2010). Examining the factor structure andhierarchical nature of the quality of life construct. American Journal of Intellectual and Developmental Disabilities, 115, 218-233. https://doi.org/10.1352/1944-7558115.3.218

Fecha de recepción: Abril de 2018.

Fecha de aceptación: Julio de 2019. 